\title{
Precise Limit in Wasserstein Distance for Conditional Empirical Measures of Dirichlet Diffusion Processes*
}

\author{
Feng-Yu Wang ${ }^{a), b)}$ \\ a) Center for Applied Mathematics, Tianjin University, Tianjin 300072, China \\ b) Department of Mathematics, Swansea University, Bay Campus, Swansea, SA1 8EN, United Kingdom
}

February 9, 2021

\begin{abstract}
Let $M$ be a $d$-dimensional connected compact Riemannian manifold with boundary $\partial M$, let $V \in C^{2}(M)$ such that $\mu(\mathrm{d} x):=\mathrm{e}^{V(x)} \mathrm{d} x$ is a probability measure, and let $X_{t}$ be the diffusion process generated by $L:=\Delta+\nabla V$ with $\tau:=\inf \left\{t \geq 0: X_{t} \in \partial M\right\}$. Consider the conditional empirical measure $\mu_{t}^{\nu}:=\mathbb{E}^{\nu}\left(\frac{1}{t} \int_{0}^{t} \delta_{X_{s}} \mathrm{~d} s \mid t<\tau\right)$ for the diffusion process with initial distribution $\nu$ such that $\nu(\partial M)<1$. Then

$$
\lim _{t \rightarrow \infty}\left\{t \mathbb{W}_{2}\left(\mu_{t}^{\nu}, \mu_{0}\right)\right\}^{2}=\frac{1}{\left\{\mu\left(\phi_{0}\right) \nu\left(\phi_{0}\right)\right\}^{2}} \sum_{m=1}^{\infty} \frac{\left\{\nu\left(\phi_{0}\right) \mu\left(\phi_{m}\right)+\mu\left(\phi_{0}\right) \nu\left(\phi_{m}\right)\right\}^{2}}{\left(\lambda_{m}-\lambda_{0}\right)^{3}}
$$

where $\nu(f):=\int_{M} f \mathrm{~d} \nu$ for a measure $\nu$ and $f \in L^{1}(\nu), \mu_{0}:=\phi_{0}^{2} \mu,\left\{\phi_{m}\right\}_{m \geq 0}$ is the eigenbasis of $-L$ in $L^{2}(\mu)$ with the Dirichlet boundary, $\left\{\lambda_{m}\right\}_{m \geq 0}$ are the corresponding Dirichlet eigenvalues, and $\mathbb{W}_{2}$ is the $L^{2}$-Wasserstein distance induced by the Riemannian metric.
\end{abstract}

AMS subject Classification: 60D05, 58J65.

Keywords: Conditional empirical measure, Dirichlet diffusion process, Wasserstein distance, eigenvalues, eigenfunctions.

*Supported in part by the National Key R\&D Program of China (No. 2020YFA0712900), NNSFC (11771326, 11831014, 11921001), and DFG through the CRC Taming uncertainty and profiting from randomness and low regularity in analysis, stochastics and their applications. 


\section{Introduction}

Let $M$ be a $d$-dimensional connected compact Riemannian manifold with a smooth boundary $\partial M$. Let $V \in C^{2}(M)$ such that $\mu(\mathrm{d} x)=\mathrm{e}^{V(x)} \mathrm{d} x$ is a probability measure on $M$, where $\mathrm{d} x$ is the Riemannian volume measure. Let $X_{t}$ be the diffusion process generated by $L:=\Delta+\nabla V$ with hitting time

$$
\tau:=\inf \left\{t \geq 0: X_{t} \in \partial M\right\} .
$$

Here, according to the convention in Riemannian geometry, the vector field $\nabla V$ is regarded as a first-order differential operator with $(\nabla V) f:=\langle\nabla V, \nabla f\rangle$ for differentiable functions $f$. Denote by $\mathscr{P}$ the set of all probability measures on $M$, and let $\mathbb{E}^{\nu}$ be the expectation taken for the diffusion process with initial distribution $\nu \in \mathscr{P}$. Consider the conditional empirical measure

$$
\mu_{t}^{\nu}:=\mathbb{E}^{\nu}\left(\frac{1}{t} \int_{0}^{t} \delta_{X_{s}} \mathrm{~d} s \mid t<\tau\right), \quad t>0, \nu \in \mathscr{P} .
$$

Since $\tau=0$ when $X_{0} \in \partial M$, to ensure $\mathbb{P}^{\nu}(\tau>t)>0$ we only consider

$$
\nu \in \mathscr{P}_{0}:=\left\{\nu \in \mathscr{P}: \nu\left(M^{\circ}\right)>0\right\}, \quad M^{\circ}:=M \backslash \partial M
$$

Let $\left\{\phi_{m}\right\}_{m \geq 0}$ be the eigenbasis in $L^{2}(\mu)$ of $-L$ with the Dirichlet boundary such that $\phi_{0}>0$ in $M^{\circ}$, and let $\left\{\lambda_{m}\right\}_{m \geq 0}$ be the associated eigenvalues listed in the increasing order counting multiplicities; that is, $\left\{\phi_{m}\right\}_{m \geq 0}$ is an orthonormal basis of $L^{2}(\mu)$ such that

$$
L \phi_{m}=-\lambda_{m} \phi_{m}, \quad m \geq 0 .
$$

Then $\mu_{0}:=\phi_{0}^{2} \mu$ is a probability measure on $M$. It is easy to see from [5, Theorem 2.1] that for any probability measure $\nu$ supported on $M^{\circ}$, we have

$$
\lim _{t \rightarrow \infty}\left\|\mu_{t}^{\nu}-\mu_{0}\right\|_{v a r}=0
$$

where $\|\cdot\|_{\text {var }}$ is the total variational norm.

In this paper, we investigate the convergence of $\mu_{t}^{\nu}$ to $\mu_{0}$ under the Wasserstein distance $\mathbb{W}_{2}$ :

$$
\mathbb{W}_{2}\left(\mu_{1}, \mu_{2}\right):=\inf _{\pi \in \mathscr{C}\left(\mu_{1}, \mu_{2}\right)}\left(\int_{M \times M} \rho(x, y)^{2} \pi(\mathrm{d} x, \mathrm{~d} y)\right)^{\frac{1}{2}}, \quad \mu_{1}, \mu_{2} \in \mathscr{P},
$$

where $\mathscr{C}\left(\mu_{1}, \mu_{2}\right)$ is the set of all probability measures on $M \times M$ with marginal distributions $\mu_{1}$ and $\mu_{2}$, and $\rho(x, y)$ is the Riemannian distance between $x$ and $y$, i.e. the length of the shortest curve on $M$ linking $x$ and $y$.

Recently, the convergence rate under $\mathbb{W}_{2}$ has been characterized in [18] for the empirical measures of the $L$-diffusion processes without boundary (i.e. $\partial M=\emptyset$ ) or with a reflecting boundary. Since in the present setting the diffusion process is killed at time $\tau$, it is reasonable to consider the conditional empirical measure $\mu_{t}^{\nu}$ given $t<\tau$. This is a counterpart to the quasi-ergodicity for the convergence of the conditional distribution $\tilde{\mu}_{t}$ of $X_{t}$ given $t<$ $\tau$. Unlike in the case without boundary or with a reflecting boundary where both the 
distribution and the empirical measure of $X_{t}$ converge to the unique invariant probability measure, in the present case the conditional distribution $\tilde{\mu}_{t}$ of $X_{t}$ given $t<\tau$ converges to $\tilde{\mu}_{0}:=\frac{\phi_{0}}{\mu\left(\phi_{0}\right)} \mu$ rather than $\mu_{0}:=\phi_{0}^{2} \mu$, and this convergence is called the quasi-ergodicity in the literature, see for instance [6] and references within.

Let $\nu(f):=\int_{M} f \mathrm{~d} \nu$ for $\nu \in \mathscr{P}$ and $f \in L^{1}(\nu)$. The main result of this paper is the following.

Theorem 1.1. For any $\nu \in \mathscr{P}_{0}$,

$$
\lim _{t \rightarrow \infty}\left\{t^{2} \mathbb{W}_{2}\left(\mu_{t}^{\nu}, \mu_{0}\right)^{2}\right\}=I:=\frac{1}{\left\{\mu\left(\phi_{0}\right) \nu\left(\phi_{0}\right)\right\}^{2}} \sum_{m=1}^{\infty} \frac{\left\{\nu\left(\phi_{0}\right) \mu\left(\phi_{m}\right)+\mu\left(\phi_{0}\right) \nu\left(\phi_{m}\right)\right\}^{2}}{\left(\lambda_{m}-\lambda_{0}\right)^{3}}>0
$$

If either $d \leq 6$ or $d \geq 7$ but $\nu=h \mu$ with $h \in L^{\frac{2 d}{d+6}}(\mu)$, then $I<\infty$.

Remark 1.1. (1) Let $X_{t}$ be the (reflecting) diffusion process generated by $L$ on $M$ where $\partial M$ may be empty. We consider the mean empirical measure $\hat{\mu}_{t}^{\nu}:=\mathbb{E}\left(\frac{1}{t} \int_{0}^{t} \delta_{X_{s}} \mathrm{~d} s\right)$, where $\nu$ is the initial distribution of $X_{t}$. Then

$$
\lim _{t \rightarrow \infty}\left\{t^{2} \mathbb{W}_{2}\left(\hat{\mu}_{t}^{\nu}, \mu_{0}\right)^{2}\right\}=\sum_{m=1}^{\infty} \frac{\left\{\nu\left(\phi_{m}\right)\right\}^{2}}{\lambda_{m}^{3}}<\infty
$$

where $\left\{\phi_{m}\right\}_{m \geq 1}$ is the eigenbasis of $-L$ in $L^{2}(\mu)$ with the Neumann boundary condition if $\partial M$ exists, $\left\{\lambda_{m}\right\}_{m \geq 1}$ are the corresponding non-trivial (Neumann) eigenvalues, and the limit is zero if and only if $\nu=\mu$. This can be confirmed by the proof of Theorem 1.1 with $\phi_{0}=1, \lambda_{0}=0$ and $\mu\left(\phi_{m}\right)=0$ for $m \geq 1$. In this case, $\mu$ is the unique invariant probability measure of $X_{t}$, so that $\hat{\mu}_{t}^{\mu}=\mu$ for $t \geq 0$ and hence the limit in (1.1) is zero for $\nu=\mu$. However, in the Dirichlet diffusion case, the conditional distribution of $\left(X_{s}\right)_{0 \leq s \leq t}$ given $t<\tau$ is no longer stationary, so that even starting from the limit distribution $\mu_{0}$ we do not have $\mu_{t}^{\mu_{0}}=\mu_{0}$ for $t>0$. This leads to a non-zero limit in Theorem 1.1 even for $\nu=\mu_{0}$.

(2) It is also interesting to investigate the convergence of $\mathbb{E}^{\nu}\left(\mathbb{W}_{2}\left(\mu_{t}, \mu_{0}\right)^{2} \mid t<\tau\right)$ for $\mu_{t}:=\frac{1}{t} \int_{0}^{t} \delta_{X_{s}} \mathrm{~d} s$, which is the counterpart to the study of [18] where the case without boundary or with a reflecting boundary is considered. According to [18], the convergence rate of $\mathbb{E}^{\nu}\left(\mathbb{W}_{2}\left(\mu_{t}, \mu_{0}\right)^{2} \mid t<\tau\right)$ will be at most $t^{-1}$, which is slower than the rate $t^{-2}$ for $\mathbb{W}_{2}\left(\mu_{t}^{\nu}, \mu_{0}\right)^{2}$ as shown in Theorem 1.1, see [15] for details, see also [16, 17] for extensions to diffusion processes on non-compact manifolds and SPDEs.

(3) Let $\nu=h \mu$. It is easy to see that $I<\infty$ is equivalent to $h \in \mathscr{D}\left((-L)^{-\frac{3}{2}}\right)$. By the Sobolev inequality, for any $p \in\left[1, \frac{d}{3}\right)$, there exists a constant $K>0$ such that

$$
\left\|(-L)^{-\frac{3}{2}} f\right\|_{L^{\frac{d p}{d-3 p}}(\mu)} \leq K\|f\|_{L^{p}(\mu)}, \quad f \in L^{p}(\mu)
$$

Taking $p=\frac{2 d}{d+6}$ which is large than 1 when $d \geq 7$, we see that $h \in L^{p}(\mu)$ implies $h \in$ $\mathscr{D}\left((-L)^{-\frac{3}{2}}\right)$ and hence $I<\infty$. So, the sharpness of the Sobolev inequality implies that of the condition $h \in L^{\frac{2 d}{d+6}}(\mu)$. 
In Section 2, we first recall some well known facts on the Dirichlet semigroup, then present an upper bound estimate on $\left\|\nabla\left(\phi_{m} \phi_{0}^{-1}\right)\right\|_{\infty}$. The latter is non-trivial when $\partial M$ is non-convex, and should be interesting by itself. With these preparations, we prove upper and lower bound estimates in Sections 3 and 4 respectively.

\section{Some preparations}

We first recall some well known facts on the Dirichlet semigroup, see for instances [4, 17, 8, 13]. Let $\left\{\phi_{m}\right\}_{m \geq 0}$ be the eigenbasis of the Dirichlet operator $L$ in $L^{2}(\mu)$, with Dirichlet eigenvalues $\left\{\lambda_{m}\right\}_{m \geq 0}$ of $-L$ listed in the increasing order counting multiplicities; that is, $\left\{\phi_{m}\right\}_{m \geq 0}$ is an orthonormal basis of $L^{2}(\mu)$ such that

$$
L \phi_{m}=-\lambda_{m} \phi_{m}, \quad m \geq 0 .
$$

For simplicity, we denote $a \preceq b$ for two positive functions $a$ and $b$ if $a \leq c b$ holds for some constant $c>0$. Then $\lambda_{0}>0$ and

$$
\left\|\phi_{m}\right\|_{\infty} \preceq \sqrt{m}, \quad m^{\frac{2}{d}} \preceq \lambda_{m}-\lambda_{0} \preceq m^{\frac{2}{d}}, \quad m \geq 1 .
$$

Let $\rho_{\partial}$ be the Riemannian distance function to the boundary $\partial M$. Then $\phi_{0}^{-1} \rho_{\partial}$ is bounded such that

$$
\left\|\phi_{0}^{-1}\right\|_{L^{p}\left(\mu_{0}\right)}<\infty, \quad p \in[1,3) .
$$

The Dirichlet heat kernel has the representation

$$
p_{t}^{D}(x, y)=\sum_{m=0}^{\infty} \mathrm{e}^{-\lambda_{m} t} \phi_{m}(x) \phi_{m}(y), \quad t>0, x, y \in M .
$$

Let $\mathbb{E}^{x}$ denote the expectation for the $L$-diffusion process starting at point $x$. Then Dirichlet diffusion semigroup generated by $L$ is given by

$$
\begin{aligned}
& P_{t}^{D} f(x):=\mathbb{E}^{x}\left[f\left(X_{t}\right) 1_{\{t<\tau\}}\right]=\int_{M} p_{t}^{D}(x, y) f(y) \mu(\mathrm{d} y) \\
& =\sum_{m=0}^{\infty} \mathrm{e}^{-\lambda_{m} t} \mu\left(\phi_{m} f\right) \phi_{m}(x), \quad t>0, f \in L^{2}(\mu) .
\end{aligned}
$$

We have

$$
\left\|P_{t}^{D}\right\|_{L^{p}(\mu) \rightarrow L^{q}(\mu)}:=\sup _{\mu\left(|f|^{p}\right) \leq 1}\left\|P_{t}^{D} f\right\|_{L^{q}(\mu)} \preceq \mathrm{e}^{-\lambda_{0} t}(1 \wedge t)^{-\frac{d(q-p)}{2 p q}}, \quad t>0, q \geq p \geq 1 .
$$

Next, let $L_{0}=L+2 \nabla \log \phi_{0}$. Then $L_{0}$ is a self-adjoint operator in $L^{2}\left(\mu_{0}\right)$ with semigroup $P_{t}^{0}:=\mathrm{e}^{t L_{0}}$ satisfying

$$
P_{t}^{0} f=\mathrm{e}^{\lambda_{0} t} \phi_{0}^{-1} P_{t}^{D}\left(f \phi_{0}\right), \quad f \in L^{2}\left(\mu_{0}\right), \quad t \geq 0 .
$$


So, $\left\{\phi_{0}^{-1} \phi_{m}\right\}_{m \geq 0}$ is an eigenbasis of $L_{0}$ in $L^{2}\left(\mu_{0}\right)$ with

$$
L_{0}\left(\phi_{m} \phi_{0}^{-1}\right)=-\left(\lambda_{m}-\lambda_{0}\right) \phi_{m} \phi_{0}^{-1}, \quad P_{t}^{0}\left(\phi_{m} \phi_{0}^{-1}\right)=\mathrm{e}^{-\left(\lambda_{m}-\lambda_{0}\right) t} \phi_{m} \phi_{0}^{-1}, \quad m \geq 0, t \geq 0 .
$$

Consequently,

$$
P_{t}^{0} f=\sum_{m=0}^{\infty} \mu_{0}\left(f \phi_{m} \phi_{0}^{-1}\right) \mathrm{e}^{-\left(\lambda_{m}-\lambda_{0}\right) t} \phi_{m} \phi_{0}^{-1}, \quad f \in L^{2}\left(\mu_{0}\right),
$$

and the heat kernel of $P_{t}^{0}$ with respect to $\mu_{0}$ is given by

$$
p_{t}^{0}(x, y)=\sum_{m=0}^{\infty}\left(\phi_{m} \phi_{0}^{-1}\right)(x)\left(\phi_{m} \phi_{0}^{-1}\right)(y) \mathrm{e}^{-\left(\lambda_{m}-\lambda_{0}\right) t}, \quad x, y \in M, t>0 .
$$

By the intrinsic ultracontractivity, see for instance [9], we have

$$
\left\|P_{t}^{0}-\mu_{0}\right\|_{L^{1}\left(\mu_{0}\right) \rightarrow L^{\infty}\left(\mu_{0}\right)}:=\sup _{\mu_{0}(|f|) \leq 1}\left\|P_{t}^{0} f-\mu_{0}(f)\right\|_{\infty} \preceq \frac{\mathrm{e}^{-\left(\lambda_{1}-\lambda_{0}\right) t}}{(1 \wedge t)^{\frac{d+2}{2}}}, \quad t>0 .
$$

Combining this with the semigroup property and the contraction of $P_{t}^{0}$ in $L^{p}(\mu)$ for any $p \geq 1$, we obtain

$$
\left\|P_{t}^{0}-\mu_{0}\right\|_{L^{p}\left(\mu_{0}\right)}:=\sup _{\mu_{0}\left(|f|^{p}\right) \leq 1}\left\|P_{t}^{0} f-\mu_{0}(f)\right\|_{L^{p}\left(\mu_{0}\right)} \preceq \mathrm{e}^{-\left(\lambda_{1}-\lambda_{0}\right) t}, \quad t \geq 0, p \geq 1 .
$$

By the interpolation theorem, (2.9) and (2.10) yield

$$
\left\|P_{t}^{0}-\mu_{0}\right\|_{L^{p}\left(\mu_{0}\right) \rightarrow L^{q}\left(\mu_{0}\right)} \preceq \mathrm{e}^{-\left(\lambda_{1}-\lambda_{0}\right) t}\{1 \wedge t\}^{-\frac{(d+2)(q-p)}{2 p q}}, \quad t>0, \infty \geq q>p \geq 1 .
$$

Since $\mu_{0}\left(\phi_{m}^{2} \phi_{0}^{-2}\right)=1$, (2.11) for $p=2$ implies

$$
\left\|\phi_{m} \phi_{0}^{-1}\right\|_{\infty}=\mathrm{e}^{\left(\lambda_{m}-\lambda_{0}\right) t}\left\|P_{t}^{0}\left(\phi_{m} \phi_{0}^{-1}\right)\right\|_{\infty} \preceq \frac{\mathrm{e}^{\left(\lambda_{m}-\lambda_{0}\right) t}}{(1 \wedge t)^{\frac{d+2}{4}}}, \quad t>0 .
$$

Taking $t=\left(\lambda_{m}-\lambda_{0}\right)^{-1}$ and applying (2.1) , we derive

$$
\left\|\phi_{m} \phi_{0}^{-1}\right\|_{\infty} \preceq m^{\frac{d+2}{2 d}}, \quad m \geq 1 .
$$

In the remainder of this section, we investigate gradient estimates on $P_{t}^{0}$ and $\phi_{m} \phi_{0}^{-1}$, which will be used in Section 4 for the study of the lower bound estimate on $\mathbb{W}_{2}\left(\mu_{t}^{\nu}, \mu_{0}\right)$. To this end, we need to estimate the Hessian tensor of $\log \phi_{0}$.

Let $N$ be the inward unit normal vector field of $\partial M$. We call $M$ (or $\partial M$ ) convex if

$$
\left\langle\nabla_{u} N, u\right\rangle=\operatorname{Hess}_{\rho_{\partial}}(u, u) \leq 0, \quad u \in T \partial M,
$$

where $\rho_{\partial}$ is the distance function to the boundary $\partial M$, and $T \partial M$ is the tangent bundle of the $(d-2)$-dimensional manifold $\partial M$. When $d=1$, the boundary $\partial M$ degenerates to a set of two end points, such that $\partial M=\emptyset$ and the condition (2.13) trivially holds; that is, $M$ is convex for $d=1$. Recall that $M^{\circ}:=M \backslash \partial M$ is the interior of $M$. 
Lemma 2.1. If $\partial M$ is convex, then there exists a constant $K_{0} \geq 0$ such that

$$
\operatorname{Hess}_{\log \phi_{0}}(u, u) \leq K_{0}|u|^{2}, \quad u \in T M^{\circ} .
$$

Proof. Since $M$ is compact with smooth boundary, there exists a constant $r_{0}>0$ such that $\rho_{\partial}$ is smooth on the set

$$
\partial_{0} M:=\left\{x \in M: \rho_{\partial}(x) \leq r_{0}\right\}
$$

Since $\phi_{0}$ is smooth and satisfies $\phi_{0} \geq c \rho_{\partial}$ for some constant $c>0$, we have $\log \left(\phi_{0} \rho_{\partial}^{-1}\right) \in$ $C_{b}^{2}\left(\partial_{0} M\right)$. So, it suffices to find a constant $c>0$ such that

$$
\operatorname{Hess}_{\log \rho_{\partial}}(u, u) \leq c|u|^{2}, \quad u \in T M^{\circ} .
$$

To this end, we fisrt estimate $\operatorname{Hess}_{\rho_{\partial}}$ on the boundary $\partial M$. For any $x \in \partial M$ and $u \in T_{x} M$, consider the orthogonal decomposition $u=u_{1}+u_{2}$, where

$$
u_{1}=\langle N, u\rangle N, \quad u_{2}:=u-u_{1} \in T \partial M
$$

Since $\left|\nabla \rho_{\partial}\right|=1$ on $\partial_{0} M$, we have

$$
\operatorname{Hess}_{\rho_{\partial}}(X, N)=\operatorname{Hess}_{\rho_{\partial}}\left(X, \nabla \rho_{\partial}\right)=\frac{1}{2}\left\langle X, \nabla\left|\nabla \rho_{\partial}\right|^{2}\right\rangle=0, \quad X \in T_{x} M .
$$

On the other hand, since $u_{2} \in T \partial M$ and $\nabla \rho_{\partial}=N$ on $\partial M$, (2.13) implies

$$
\operatorname{Hess}_{\rho_{\partial}}\left(u_{2}, u_{2}\right)=\left\langle\nabla_{u_{2}} N, u_{2}\right\rangle \leq 0 .
$$

Combining this with (2.15) we obtain

$$
\operatorname{Hess}_{\rho_{\partial}}(u, u)=\langle N, u\rangle^{2} \operatorname{Hess}_{\rho_{\partial}}(N, N)+2\langle N, u\rangle \operatorname{Hess}_{\rho_{\partial}}\left(u_{2}, N\right)+\operatorname{Hess}_{\rho_{\partial}}\left(u_{2}, u_{2}\right) \leq 0
$$

for $u \in \cup_{x \in \partial M} T_{x} M$. Since Hess $\rho_{\partial \partial}$ is smooth on the compact set $\partial_{0} M$, this implies

$$
\operatorname{Hess}_{\rho_{\partial}}(u, u) \leq c|u|^{2} \rho_{\partial}(x), \quad x \in M, u \in T_{x} M
$$

for some constant $c>0$. Then the desired estimate (2.14) follows from

$$
\operatorname{Hess}_{\log \rho_{\partial}}(u, u)=\rho_{\partial}^{-1} \operatorname{Hess}_{\rho_{\partial}}(u, u)-\rho_{\partial}^{-2}\left\langle\nabla \rho_{\partial}, u\right\rangle^{2} \leq c|u|^{2}, \quad u \in T M^{\circ} .
$$

By Lemma 2.1, when $\partial M$ is convex, there exists a constant $K \geq 0$ such that

$$
\text { Ric }-\operatorname{Hess}_{V+2} \log \phi_{0} \geq-K \text {. }
$$

Since the diffusion process generated by $L_{0}:=\Delta+\nabla\left(V+2 \log \phi_{0}\right)$ is non-explosive in $M^{\circ}$, by (2.16) and Bakry-Emery's semigroup calculus, (see for instance [3] or [13, Theorem 2.3.3]), we have

$$
\left|\nabla P_{t}^{0} g\right| \leq \mathrm{e}^{K t} P_{t}^{0}|\nabla g|, \quad t \geq 0, g \in C_{b}^{1}(M)
$$


and for any $p>1$, there exists a constant $c(p)>0$ such that

$$
\begin{aligned}
\left|\nabla P_{t}^{0} g\right|^{2} & \leq \frac{2 K\left\{\left(P_{t}^{0}|g|^{p \wedge 2}\right)\left(P_{t}^{0}|g|\right)^{(2-p)^{+}}-\left(P_{t}^{0}|g|\right)^{2}\right\}}{(p \wedge 2)(p \wedge 2-1)\left(1-\mathrm{e}^{-2 K t}\right)} \\
& \leq \frac{c(p)}{1 \wedge t}\left(P_{t}^{0}|g|^{p}\right)^{\frac{2}{p}}, \quad t>0, g \in \mathscr{B}_{b}(M)
\end{aligned}
$$

When $\partial M$ is non-convex, we take as in [12] a conformal change of metric to make it convex under the new metric. More precisely, we have the following result.

Lemma 2.2. There exists a function $1 \leq \phi \in C_{b}^{\infty}(M)$ such that $\partial M$ is convex under the metric $\langle\cdot, \cdot\rangle_{\phi}:=\phi^{-2}\langle\cdot, \cdot\rangle$. Moreover, there exists a smooth vector field $Z_{\phi}$ on $M$ such that

$$
L_{0}=\phi^{-2} \Delta^{\phi}+Z_{\phi}+2 \phi^{-1} \nabla^{\phi} \log \phi_{0}
$$

where $\nabla^{\phi}$ and $\Delta^{\phi}$ are the gradient and Lapalce-Beltrami operators induced by $\langle\cdot, \cdot\rangle_{\phi}$ respectively.

Proof. let $\delta>0$ such that the second fundamental form of $\partial M$ is bounded below by $-\delta$. Take $1 \leq \phi \in C_{b}^{\infty}(M)$ such that $\phi=1+\delta \rho_{\partial}$ in a neighborhood of $\partial M$ in which the distance function $\rho_{\partial}$ to $\partial M$ is smooth. By [14, Lemma 2.1](see also [12]), $\partial M$ is convex under the metric $\langle\cdot, \cdot\rangle_{\phi}:=\phi^{-2}\langle\cdot, \cdot\rangle$. Next, according to the proof of [14, Lemma 2.2], there exists a smooth vector field $Z_{\phi}$ on $M$ such that (2.19) holds. by

Let $1 \leq \phi \in C_{b}^{\infty}(M)$ be as in Lemma 2.2. and let $P_{t}^{\phi}$ be the diffusion semigroup generated

$$
L^{\phi}:=\phi L_{0}=\phi^{-1} \Delta^{\phi}+\phi Z_{\phi}+2 \nabla^{\phi} \log \phi_{0}
$$

We have the following result.

Lemma 2.3. Let $1 \leq \phi \in C_{b}^{\infty}(M)$ be as in Lemma 2.2.

(1) For any $q \in(1, \infty]$, there exists a constant $c(q)>0$ such that

$$
\left|\nabla^{\phi} P_{t}^{\phi} f\right|_{\phi} \leq \frac{c(q)}{\sqrt{t}}\left(P_{t}^{\phi}|f|^{q}\right)^{\frac{1}{q}}, \quad t>0, f \in C_{b}^{1}(M) .
$$

Moreover, there exists a constant $K>0$ such that

$$
\left|\nabla^{\phi} P_{t}^{\phi} f\right|_{\phi} \leq \mathrm{e}^{K t} P_{t}^{\phi}\left|\nabla^{\phi} f\right|_{\phi}, \quad t>0, f \in C_{b}^{1}(M) .
$$

(2) There exists a constant $c>0$ such that

$$
\left\|P_{t}^{\phi}\right\|_{L^{p}\left(\mu_{0}\right) \rightarrow L^{\infty}\left(\mu_{0}\right)} \leq c(1 \wedge t)^{-\frac{d+2}{2 p}}, \quad t>0, p \in[1, \infty]
$$


Proof. (1) Since $\partial M$ is convex under the metric $\langle\cdot, \cdot\rangle_{\phi}$, by Lemma 2.1, we find a constant $K_{0}^{\phi}>0$ such that

$$
2 \operatorname{Hess}_{\log \phi_{0}}^{\phi}(u, u) \leq K_{0}^{\phi}|u|_{\phi}^{2}, \quad u \in T M^{\circ}
$$

where $\operatorname{Hess}^{\phi}$ is the Hessian tensor induced by the metric $\langle\cdot, \cdot\rangle_{\phi}$. Since the operator $A^{\phi}:=$ $\phi^{-1} \Delta^{\phi}+\phi Z_{\phi}$ is a $C^{2}$-smooth strictly elliptic second order differential operator on the compact manifold $M$, it has bounded below Bakry-Emery curvature; that is, there exists a constant $K_{1}^{\phi}>0$ such that

$$
A^{\phi}\left|\nabla^{\phi} f\right|_{\phi}^{2}-2\left\langle\nabla^{\phi} A^{\phi} f, \nabla^{\phi} f\right\rangle_{\phi} \geq-K_{1}^{\phi}\left|\nabla^{\phi} f\right|_{\phi}^{2}, \quad f \in C^{\infty}(M) .
$$

Combining this with (2.23) we obtain

$$
L^{\phi}\left|\nabla^{\phi} f\right|_{\phi}^{2}-2\left\langle\nabla^{\phi} L^{\phi} f, \nabla^{\phi} f\right\rangle_{\phi} \geq-\left(K_{0}^{\phi}+K_{1}^{\phi}\right)\left|\nabla^{\phi} f\right|_{\phi}^{2}=:-K^{\phi}\left|\nabla^{\phi} f\right|_{\phi}^{2}, \quad f \in C^{\infty}\left(M^{\circ}\right),
$$

which means that the Bakry-Emery curvature of $L^{\phi}$ is bounded below by $-K^{\phi}$. By the same reason leading to (2.17) and (2.18), this implies (2.20) and (2.21).

(2) To estimate $\left\|P_{t}^{\phi}\right\|_{L^{p}\left(\mu_{0}\right) \rightarrow L^{\infty}\left(\mu_{0}\right)}$, we make use of [10, Theorem 4.5(b)] or [11, Theorem 3.3.15(2)], which says that (2.9) implies the super Poincaré inequality

$$
\mu_{0}\left(f^{2}\right) \leq r \mu_{0}\left(|\nabla f|^{2}\right)+\beta\left(1+r^{-\frac{d+2}{2}}\right) \mu_{0}(|f|)^{2}, \quad r>0, f \in C_{b}^{1}(M)
$$

for some constant $\beta>0$. Let $\mu^{\phi}=\frac{\phi^{-1} \mu_{0}}{\mu_{0}\left(\phi^{-1}\right)}$. By $L^{\phi}=\phi L_{0}$ we obtain

$\mathscr{E} \phi(f, g):=-\int_{M} f L^{\phi} g \mathrm{~d} \mu^{\phi}=-\frac{1}{\mu_{0}\left(\phi^{-1}\right)} \int_{M} f L_{0} g \mathrm{~d} \mu_{0}=\frac{1}{\mu\left(\phi^{-1}\right)} \mu_{0}(\langle\nabla f, \nabla g\rangle), \quad f, g \in C_{b}^{2}(M)$.

Then the above super Poincaré inequality implies

$$
\mu^{\phi}\left(f^{2}\right) \leq r \mathscr{E}^{\phi}(f, f)+\beta^{\prime}\left(1+r^{-\frac{d+2}{2}}\right) \mu^{\phi}(|f|)^{2}, \quad f \in C_{b}^{1}(M)
$$

for some constant $\beta^{\prime}>0$. Using [10, Theorem 4.5(b)] or [11, Theorem 3.3.15(2)] again, this implies

$$
\left\|P_{t}^{\phi}\right\|_{L^{p}\left(\mu^{\phi}\right) \rightarrow L^{\infty}\left(\mu^{\phi}\right)} \leq \kappa(1 \wedge t)^{-\frac{d+2}{2 p}}, \quad t>0, p \in[1, \infty]
$$

for some constant $\kappa>0$. Noting that

$$
\|\phi\|_{\infty}^{-1} \mu_{0} \leq \mu^{\phi} \leq\|\phi\|_{\infty} \mu_{0}
$$

we find a constant $c>0$ such that (2.22) holds.

Lemma 2.4. For any $p \in(1, \infty]$ and $q \in(1, p)$, there exists a constant $c>0$ such that for any $f \in D\left(L_{0}\right)$,

$$
\left\|\nabla P_{t}^{0} f\right\|_{\infty} \leq c \mathrm{e}^{-\lambda_{0} t}\left\{(1 \wedge t)^{-\frac{1}{2}-\frac{d+2}{2 p}}\|f\|_{L^{p}\left(\mu_{0}\right)}+(1 \wedge t)^{\frac{1}{2}-\frac{q(d+2)}{2 p}}\left\|L_{0} f\right\|_{L^{p}\left(\mu_{0}\right)}\right\}, \quad t>0 .
$$

Consequently, there exists a constant $c>0$ such that

$$
\left\|\nabla\left(\phi_{m} \phi_{0}^{-1}\right)\right\|_{\infty} \leq c m^{\frac{d+4}{2 d}}, \quad m \geq 1
$$


Proof. (a) By the semigroup property and the $L^{p}\left(\mu_{0}\right)$ contraction of $P_{t}^{0}$, for the proof of (2.24) it suffices to consider $t \in(0,1]$. Since $1 \leq \phi \in C_{b}^{\infty}(M)$, we have $\mathscr{D}\left(L_{0}\right)=\mathscr{D}\left(L^{\phi}\right)$ and

$$
P_{t}^{0} f=P_{t}^{\phi} f-\int_{0}^{t} P_{s}^{\phi}\left\{(\phi-1) P_{t-s}^{0} L_{0} f\right\} \mathrm{d} s, \quad t \geq 0, f \in \mathscr{D}\left(L_{0}\right) .
$$

Next, by (2.20) and (2.22), we obtain

$$
\begin{aligned}
& \left\|\nabla P_{t}^{\phi} f\right\|_{\infty}=\left\|\nabla P_{t / 2}^{\phi}\left(P_{t / 2}^{\phi} f\right)\right\|_{\infty} \\
& \preceq t^{-\frac{1}{2}}\left\|P_{t / 2}^{\phi} f\right\|_{\infty} \preceq t^{-\frac{1}{2}-\frac{d+2}{2 p}}\|f\|_{L^{p}\left(\mu_{0}\right)}, \quad t \in(0,1] .
\end{aligned}
$$

Combining this with (2.11) and (2.20) leads to

$$
\begin{aligned}
& \int_{0}^{t}\left\|\nabla P_{s}^{\phi}\left\{(\phi-1) P_{t-s}^{0} L_{0} f\right\}\right\|_{\infty} \mathrm{d} s \preceq \int_{0}^{t} s^{-\frac{1}{2}}\left\|\left\{P_{s}^{\phi}\left|P_{t-s}^{0} L_{0} f\right|^{q}\right\}^{\frac{1}{q}}\right\|_{\infty} \mathrm{d} s \\
& \preceq \int_{0}^{\frac{t}{2}} s^{-\frac{1}{2}}\left\|P_{t-s}^{0} L_{0} f\right\|_{\infty} \mathrm{d} s+\int_{\frac{t}{2}}^{t} s^{-\frac{1}{2}}\left\|\left\{P_{s}^{\phi}\left|P_{t-s}^{0} L_{0} f\right|^{q}\right\}^{\frac{1}{q}}\right\|_{\infty} \mathrm{d} s \\
& \preceq \int_{0}^{\frac{t}{2}} s^{-\frac{1}{2}}\left\|P_{t-s}^{0}\right\|_{L^{p}\left(\mu_{0}\right) \rightarrow L^{\infty}\left(\mu_{0}\right)}\left\|L_{0} f\right\|_{L^{p}\left(\mu_{0}\right)} \mathrm{d} s+\int_{\frac{t}{2}}^{t} s^{-\frac{1}{2}}\left\|P_{s}^{\phi}\right\|_{L^{\frac{p}{q}}\left(\mu_{0}\right) \rightarrow L^{\infty}\left(\mu_{0}\right)}\left\|L_{0} f\right\|_{L^{p}\left(\mu_{0}\right)} \\
& \preceq t^{\frac{1}{2}-\frac{q(d+2)}{2 p}}\left\|L_{0} f\right\|_{L^{p}\left(\mu_{0}\right)} .
\end{aligned}
$$

Substituting this and (2.27) into (2.26), we prove (2.24).

(b) Applying (2.24) to $p=\infty, f=\phi_{m} \phi_{0}^{-1}, t=\left(\lambda_{m}-\lambda_{0}\right)^{-1}$ and using (2.6)), we obtain

$$
\mathrm{e}^{-1}\left\|\nabla\left(\phi_{m} \phi_{0}^{-1}\right)\right\|_{\infty} \preceq\left(\lambda_{m}-\lambda_{0}\right)^{\frac{1}{2}}\left\|\phi_{m} \phi_{0}^{-1}\right\|_{\infty}, \quad m \geq 1 .
$$

This together with (2.1) and (2.12) implies (2.25) for some constant $c>0$.

\section{Upper bound estimate}

According to [18, Lemma 2.3], we have

$$
\mathbb{W}_{2}\left(\mu_{t}^{\nu}, \mu_{0}\right)^{2} \leq \int_{M} \frac{\left|\nabla L_{0}^{-1}\left(h_{t}^{\nu}-1\right)\right|^{2}}{\mathscr{M}\left(h_{t}^{\nu}, 1\right)} \mathrm{d} \mu_{0} a
$$

where

$$
h_{t}^{\nu}:=\frac{\mathrm{d} \mu_{t}^{\nu}}{\mathrm{d} \mu_{0}}, \quad \mathscr{M}(a, b):=1_{\{a \wedge b>0\}} \frac{a-b}{\log a-\log b} .
$$

So, to investigate the upper bound estimate, we first calculate $h_{t}^{\nu}$.

By (2.8), we have

$$
\psi_{s}^{\nu}:=\int_{M} \phi_{0}(x) p_{s}^{0}(x, \cdot) \nu(\mathrm{d} x)=\nu\left(\phi_{0}\right)+\sum_{m=1}^{\infty} \nu\left(\phi_{m}\right) \mathrm{e}^{-\left(\lambda_{m}-\lambda_{0}\right) s} \phi_{m} \phi_{0}^{-1}, \quad s>0 .
$$


Next, (2.5) and (2.8) imply

$$
\nu\left(P_{s}^{D} f\right)=\mathrm{e}^{-\lambda_{0} s} \nu\left(\phi_{0} P_{s}^{0}\left(f \phi_{0}^{-1}\right)\right)=\mathrm{e}^{-\lambda_{0} s} \int_{M} \psi_{s}^{\nu} \phi_{0}^{-1} f \mathrm{~d} \mu_{0}, \quad f \in \mathscr{B}^{+}(M),
$$

where $\mathscr{B}^{+}(M)$ is the class of nonnegative measurable functions on $M$. Moreover, for any $t \geq s>0$, by the Markov property, (2.3), (2.5) and (3.3), we obtain

$$
\begin{aligned}
& \int_{M} f \mathrm{~d} \mathbb{E}^{\nu}\left[\delta_{X_{s}} 1_{\{t<\tau\}}\right]=\mathbb{E}^{\nu}\left[f\left(X_{s}\right) 1_{\{s<\tau\}}\left(P_{t-s}^{D} 1\right)\left(X_{s}\right)\right]=\nu\left(P_{s}^{D}\left\{f P_{t-s}^{D} 1\right\}\right) \\
& =\mathrm{e}^{-\lambda_{0} t} \int_{M}\left(\psi_{s}^{v} P_{t-s}^{0} \phi_{0}^{-1}\right) f \mathrm{~d} \mu_{0}, \quad f \in \mathscr{B}^{+}(M) .
\end{aligned}
$$

Then

$$
\frac{\mathrm{d} \mathbb{E}^{\nu}\left[\delta_{X_{s}} 1_{\{t<\tau\}}\right]}{\mathrm{d} \mu_{0}}=\mathrm{e}^{-\lambda_{0} t} \psi_{s}^{v} P_{t-s}^{0} \phi_{0}^{-1}
$$

Noting that (3.3) implies

$$
\mathbb{E}^{\nu}\left[1_{\{t<\tau\}}\right]=\nu\left(P_{t}^{D} 1\right)=\mathrm{e}^{-\lambda_{0} t} \mu_{0}\left(\psi_{t}^{\nu} \phi_{0}^{-1}\right)=\mathrm{e}^{-\lambda_{0} t} \nu\left(\phi_{0} P_{t}^{0} \phi_{0}^{-1}\right),
$$

we arrive at

$$
\begin{aligned}
& h_{t}^{\nu}:=\frac{\mathrm{d} \mu_{t}^{\nu}}{\mathrm{d} \mu_{0}}=\frac{1}{t \mathbb{E}^{\nu} 1_{\{t<\tau\}}} \int_{0}^{t} \frac{\mathrm{d} \mathbb{E}^{\nu}\left[\delta_{X_{s}} 1_{\{t<\tau\}}\right]}{\mathrm{d} \mu_{0}} \mathrm{~d} s=1+\rho_{t}^{\nu}, \\
& \rho_{t}^{\nu}:=\frac{1}{t \nu\left(\phi_{0} P_{t}^{0} \phi_{0}^{-1}\right)} \int_{0}^{t}\left\{\psi_{s}^{\nu} P_{t-s}^{0} \phi_{0}^{-1}-\nu\left(\phi_{0} P_{t}^{0} \phi_{0}^{-1}\right)\right\} \mathrm{d} s .
\end{aligned}
$$

By (2.11), $\left\|\phi_{0}\right\|_{\infty}<\infty$ and $\left\|\phi_{0}^{-1}\right\|_{L^{2}\left(\mu_{0}\right)}=1$, we find a constant $c>0$ such that

$$
\begin{aligned}
& \left|\nu\left(\phi_{0} P_{t}^{0} \phi_{0}^{-1}\right)-\nu\left(\phi_{0}\right) \mu\left(\phi_{0}\right)\right| \leq \nu\left(\phi_{0}\right)\left\|P_{t}^{0} \phi_{0}^{-1}-\mu_{0}\left(\phi_{0}^{-1}\right)\right\|_{\infty} \\
& \leq c \mathrm{e}^{-\left(\lambda_{1}-\lambda_{0}\right) t}, \quad t \geq 1, \nu \in \mathscr{P}_{0} .
\end{aligned}
$$

Due to the lack of simple representation of the product $\psi_{s}^{\nu} P_{t-s}^{0} \phi_{0}^{-1}$ in terms of the eigenbasis $\left\{\phi_{m} \phi_{0}^{-1}\right\}_{m \geq 0}$, it is inconvenient to estimate the upper bound in (3.1). To this end, below we reduce this product to a linear combination of $\psi_{s}^{\nu}$ and $P_{t-s}^{0} \phi_{0}^{-1}$, for which the spectral representation works. Write

$$
\begin{aligned}
& \psi_{s}^{\nu} P_{t-s}^{0} \phi_{0}^{-1}-\nu\left(\phi_{0} P_{t}^{0} \phi_{0}^{-1}\right)=I_{1}(s)+I_{2}(s), \\
& I_{1}(s):=\left\{\psi_{s}^{\nu}-\nu\left(\phi_{0}\right)\right\} \cdot\left\{P_{t-s}^{0} \phi_{0}^{-1}-\mu\left(\phi_{0}\right)\right\}+\nu\left(\phi_{0}\left\{\mu\left(\phi_{0}\right)-P_{t}^{0} \phi_{0}^{-1}\right\}\right), \\
& I_{2}(s):=\mu\left(\phi_{0}\right)\left\{\psi_{s}^{\nu}-\nu\left(\phi_{0}\right)\right\}+\nu\left(\phi_{0}\right)\left\{P_{t-s}^{0} \phi_{0}^{-1}-\mu\left(\phi_{0}\right)\right\} .
\end{aligned}
$$

By (2.7), (2.8) and (3.2), we have

$$
\begin{aligned}
& P_{t-s}^{0} \phi_{0}^{-1}-\mu\left(\phi_{0}\right)=\sum_{m=1}^{\infty} \mu\left(\phi_{m}\right) \mathrm{e}^{-\left(\lambda_{m}-\lambda_{0}\right)(t-s)} \phi_{m} \phi_{0}^{-1}, \\
& \psi_{s}^{\nu}-\nu\left(\phi_{0}\right)=\sum_{m=1}^{\infty} \nu\left(\phi_{m}\right) \mathrm{e}^{-\left(\lambda_{m}-\lambda_{0}\right) s} \phi_{m} \phi_{0}^{-1}, \quad t>s>0 .
\end{aligned}
$$


Then

$$
\begin{aligned}
\rho_{t}^{\nu} & =\tilde{\rho}_{t}^{\nu}+\frac{1}{t \nu\left(\phi_{0} P_{t}^{0} \phi_{0}^{-1}\right)} \int_{0}^{t} I_{1}(s) \mathrm{d} s-A_{t} \\
\tilde{\rho}_{t}^{\nu} & :=\frac{1}{t \nu\left(\phi_{0} P_{t}^{0} \phi_{0}^{-1}\right)} \sum_{m=1}^{\infty} \frac{\mu\left(\phi_{0}\right) \nu\left(\phi_{m}\right)+\nu\left(\phi_{0}\right) \mu\left(\phi_{m}\right)}{\lambda_{m}-\lambda_{0}} \phi_{m} \phi_{0}^{-1} \\
A_{t} & :=\frac{1}{t \nu\left(\phi_{0} P_{t}^{0} \phi_{0}^{-1}\right)} \sum_{m=1}^{\infty} \frac{\left\{\mu\left(\phi_{0}\right) \nu\left(\phi_{m}\right)+\nu\left(\phi_{0}\right) \mu\left(\phi_{m}\right)\right\} \mathrm{e}^{-\left(\lambda_{m}-\lambda_{0}\right) t}}{\lambda_{m}-\lambda_{0}} \phi_{m} \phi_{0}^{-1} .
\end{aligned}
$$

Since $\rho_{t}^{\nu} \in L^{1}\left(\mu_{0}\right)$, the following lemma implies $\tilde{\rho}_{t}^{\nu} \in L^{1}\left(\mu_{0}\right)$ for $t>0$.

Lemma 3.1. For any $t_{0}>0$, there exists a constant $c>0$ such that

$$
\mu_{0}\left(\left|\rho_{t}^{\nu}-\tilde{\rho}_{t}^{\nu}\right|\right) \leq c\|h\|_{L^{2}(\mu)} \mathrm{e}^{-\left(\lambda_{1}-\lambda_{0}\right) t}, \quad t \geq t_{0}, \nu=h \mu \in \mathscr{P}_{0} .
$$

Proof. By (2.1) and (2.12), for any $t_{0}>0$ we have

$$
\sum_{m=1}^{\infty}\left\|\phi_{m}\right\|_{\infty} \mathrm{e}^{-\left(\lambda_{m}-\lambda_{0}\right) t} \preceq \mathrm{e}^{-\left(\lambda_{1}-\lambda_{0}\right) t}, \quad t \geq t_{0}
$$

Combining this with (3.8) and (3.5), and noting that $\left\|h \phi_{0}^{-1}\right\|_{L^{2}\left(\mu_{0}\right)}=\|h\|_{L^{2}(\mu)}$, it suffices to show that

$$
B:=\frac{1}{t} \int_{0}^{t}\left\|\left\{\psi_{s}^{\nu}-\nu\left(\phi_{0}\right)\right\} \cdot\left\{P_{t-s}^{0} \phi_{0}^{-1}-\mu\left(\phi_{0}\right)\right\}\right\|_{L^{1}\left(\mu_{0}\right)} \mathrm{d} s \preceq\|h\|_{L^{2}(\mu)} \mathrm{e}^{-\left(\lambda_{1}-\lambda_{0}\right) t}, \quad t \geq t_{0} .
$$

Since $\left\|\phi_{0}^{-1}\right\|_{L^{2}\left(\mu_{0}\right)}=1$ and $\psi_{s}^{\nu}=P_{0}^{s}\left(h \phi_{0}^{-1}\right)$ for $\nu=h \mu$, (2.10) yields that

$$
\begin{aligned}
& B \leq \frac{1}{t} \int_{0}^{t}\left\|P_{t-s}^{0} \phi_{0}^{-1}-\mu_{0}\left(\phi_{0}^{-1}\right)\right\|_{L^{2}\left(\mu_{0}\right)}\left\|P_{s}^{0}\left(h \phi_{0}^{-1}\right)-\mu_{0}\left(h \phi_{0}^{-1}\right)\right\|_{L^{2}\left(\mu_{0}\right)} \mathrm{d} s \\
& \leq \frac{1}{t} \int_{0}^{t}\left\|P_{t-s}^{0}-\mu_{0}\right\|_{L^{2}\left(\mu_{0}\right)}\left\|P_{s}^{0}-\mu_{0}\right\|_{L^{2}\left(\mu_{0}\right)}\|h\|_{L^{2}(\mu)} \mathrm{d} s \\
& \preceq\|h\|_{L^{2}(\mu)} \mathrm{e}^{-\left(\lambda_{1}-\lambda_{0}\right) t}, \quad t \geq t_{0} .
\end{aligned}
$$

Lemma 3.2. For any $\alpha>0$, there exist constants $c_{0}, t_{0}>0$ such that

$$
\tilde{\rho}_{t}^{\nu} \geq-\frac{c_{0}}{\nu\left(\phi_{0}\right) t}, \quad t \geq t_{0}, \quad \nu \in \mathscr{P}_{0}, \nu \in \mathscr{P}_{0}
$$

Consequently, if $\nu=h \mu$ with $h \in L^{2}(\mu)$, then $\tilde{\mu}_{t}^{\nu}:=\left(1+\tilde{\rho}_{t}^{\nu}\right) \mu_{0}$ is a probability measure for $t>t_{0}\left(1+c_{0}\right)$. 
Proof. By Lemma 3.1, if $\nu=h \mu$ with $h \in L^{2}(\mu)$, we have $\tilde{\rho}_{t}^{\nu} \in L^{1}\left(\mu_{0}\right)$ for $t>0$, and it is easy to see that $\mu_{0}\left(\tilde{\rho}_{t}^{\nu}\right)=0$. Since (3.12) implies $1+\tilde{\rho}_{t}^{\nu}>0$ for $t>t_{0}\left(1+c_{0}\right)$, $\tilde{\mu}_{t}^{\nu}$ is a probability measure. It remains to prove (3.12).

By (3.5) and (3.8), it suffices to find a constant $c_{1}>0$ such that

$$
g:=\sum_{m=1}^{\infty} \frac{\mu\left(\phi_{0}\right) \nu\left(\phi_{m}\right)+\nu\left(\phi_{0}\right) \mu\left(\phi_{m}\right)}{\lambda_{m}-\lambda_{0}} \phi_{m} \phi_{0}^{-1} \geq-c_{1} .
$$

By (2.1) and (2.12), we have

$$
\left\|P_{1}^{0} g\right\|_{\infty} \leq c_{2}:=\sum_{m=1}^{\infty} \frac{2\left\|\phi_{0}\right\|_{\infty}\left\|\phi_{m}\right\|_{\infty}\left\|\phi_{m} \phi_{0}^{-1}\right\|_{\infty}}{\left(\lambda_{m}-\lambda_{0}\right) \mathrm{e}^{\lambda_{m}-\lambda_{0}}}<\infty
$$

Next, by (3.7) and the same formula for $\mu=\nu$, we obtain

$$
P_{s}^{0} g=\left(-L_{0}\right)^{-1}\left\{\mu\left(\phi_{0}\right)\left(\psi_{s}^{\nu}-\nu\left(\phi_{0}\right)\right)+\nu\left(\phi_{0}\right)\left(\psi_{s}^{\mu}-\mu\left(\phi_{0}\right)\right)\right\}=\left(-L_{0}\right)^{-1} g_{s}, \quad s>0,
$$

where by $\phi_{0}, \psi_{s}^{\nu}, \psi_{s}^{\mu} \geq 0$,

$$
g_{s}:=\mu\left(\phi_{0}\right)\left(\psi_{s}^{\nu}-\nu\left(\phi_{0}\right)\right)+\nu\left(\phi_{0}\right)\left(\psi_{s}^{\mu}-\mu\left(\phi_{0}\right)\right) \geq-2 \mu\left(\phi_{0}\right) \nu\left(\phi_{0}\right) \geq-2 \nu\left(\phi_{0}\right), \quad s>0 .
$$

This together with (3.15) yields

$$
-L_{0} P_{s}^{0} g \geq-2 \nu\left(\phi_{0}\right), \quad s>0 .
$$

Therefore, it follows from (3.14) that

$$
g=P_{1}^{0} g-\int_{0}^{1} L_{0} P_{r}^{0} g \mathrm{~d} r \geq-c_{2}-2 \nu\left(\phi_{0}\right) \geq-c_{2}-2\left\|\phi_{0}\right\|_{\infty} .
$$

So, (3.13) holds for $c_{1}=c_{2}+2\left\|\phi_{0}\right\|_{\infty}$.

Lemma 3.3. There exist constants $c, t_{0}>0$ such that for any $t \geq t_{0}$, and any $\nu \in \mathscr{P}_{0}$ with $\nu=h \mu$ such that $h \in L^{2}(\mu)$, we have $\tilde{\mu}_{t}^{\nu} \in \mathscr{P}_{0}$ and

$$
t^{2} \mathbb{W}_{2}\left(\tilde{\mu}_{t}^{\nu}, \mu_{0}\right)^{2} \leq \frac{1+c t^{-1}}{\left\{\mu\left(\phi_{0}\right) \nu\left(\phi_{0}\right)\right\}^{2}} \sum_{m=1}^{\infty} \frac{\left\{\nu\left(\phi_{0}\right) \mu_{0}\left(\phi_{m}\right)+\mu\left(\phi_{0}\right) \nu\left(\phi_{m}\right)\right\}^{2}}{\left(\lambda_{m}-\lambda_{0}\right)^{3}}
$$

Proof. By Lemma 3.2, there exist constants $c, t_{0}>0$ such that $\tilde{\mu}_{t}^{\nu} \in \mathscr{P}_{0}$ for $t \geq t_{0}$, and

$$
\mathscr{M}\left(1+\tilde{\rho}_{t}^{\nu}, 1\right) \geq 1 \wedge\left(1+\tilde{\rho}_{t}^{\nu}\right) \geq \frac{1}{1+c t^{-1}}, \quad t \geq t_{0}
$$

So, [18, Lemma 2.3] implies

$$
\mathbb{W}_{2}\left(\tilde{\mu}_{t}^{\nu}, \mu_{0}\right)^{2} \leq \int_{M} \frac{\left|\nabla L_{0}^{-1} \tilde{\rho}_{t}^{\nu}\right|^{2}}{\mathscr{M}\left(1+\tilde{\rho}_{t}^{\nu}, 1\right)} \mathrm{d} \mu_{0} \leq\left(1+c t^{-1}\right) \mu_{0}\left(\left|\nabla L_{0}^{-1} \tilde{\rho}_{t}^{\nu}\right|^{2}\right), \quad t \geq t_{0}
$$

Next, (2.6) and (3.8) yield

$$
t^{2} \mu_{0}\left(\left|\nabla L_{0}^{-1} \tilde{\rho}_{t}^{\nu}\right|^{2}\right)=\frac{1}{\left\{\nu\left(\phi_{0} P_{t}^{0} \phi_{0}^{-1}\right)\right\}^{2}} \sum_{m=1}^{\infty} \frac{\left\{\mu\left(\phi_{0}\right) \nu\left(\phi_{m}\right)+\nu\left(\phi_{0}\right) \mu\left(\phi_{m}\right)\right\}^{2}}{\left(\lambda_{m}-\lambda_{0}\right)^{3}} .
$$

Combining this with (3.5) and (3.17), we finish the proof. 
We are now ready to prove the following result.

Proposition 3.4. For any $\nu \in \mathscr{P}_{0}$,

$$
\limsup _{t \rightarrow \infty}\left\{t^{2} \mathbb{W}_{2}\left(\mu_{t}^{\nu}, \mu_{0}\right)^{2}\right\} \leq I
$$

Proof. (1) We first consider $\nu=h \mu$ with $h \in L^{2}(\mu)$. Let $D$ be the diameter of $M$. By Lemma [3.1, there exist constants $c_{1}, t_{0}>0$ such that $\tilde{\mu}_{t}^{\nu}$ is probability measure for $t \geq t_{0}$ and

$$
\mathbb{W}_{2}\left(\mu_{t}^{\nu}, \tilde{\mu}_{t}^{\nu}\right)^{2} \leq D^{2}\left\|\mu_{t}^{\nu}-\tilde{\mu}_{t}^{\nu}\right\|_{v a r}=D^{2} \mu_{0}\left(\left|\rho_{t}^{\nu}-\tilde{\rho}_{t}^{\nu}\right|\right) \leq c_{1}\|h\|_{L^{2}(\mu)} \mathrm{e}^{-\left(\lambda_{1}-\lambda_{0}\right) t}, \quad t \geq t_{0} .
$$

Combining this with Lemma 3.3 and the triangle inequality of $\mathbb{W}_{2}$, we obtain

$$
t^{2} \mathbb{W}_{2}\left(\mu_{t}^{\nu}, \mu_{0}\right)^{2} \leq\left(1+\delta^{-1}\right) c_{1} t^{2} \mathrm{e}^{-\left(\lambda_{1}-\lambda_{0}\right) t}\|h\|_{L^{2}(\mu)}+(1+\delta)\left(1+c t^{-1}\right) I, \quad \delta>0 .
$$

(2) In general, we may go back to the first situation by shifting a small time $\varepsilon>0$. More precisely, by the Markov property, (2.3), (2.5) and (3.2), for any $f \in \mathscr{B}_{b}(M)$ and $t \geq s \geq \varepsilon>0$, we have

$$
\begin{aligned}
& \mathbb{E}^{\nu}\left[f\left(X_{s}\right) 1_{\{t<\tau\}}\right]=\mathbb{E}^{\nu}\left[1_{\{\varepsilon<\tau\}} \mathbb{E}^{X_{\varepsilon}}\left(f\left(X_{s-\varepsilon}\right) 1_{\{t-\varepsilon<\tau\}}\right)\right] \\
& =\int_{M} p_{\varepsilon}^{D}(x, y) \mathbb{E}^{y}\left[f\left(X_{s-\varepsilon}\right) 1_{\{t-\varepsilon<\tau\}}\right] \nu(\mathrm{d} x) \mu(\mathrm{d} y) \\
& =\mathrm{e}^{-\lambda_{0} \varepsilon} \int_{M}\left(\psi_{\varepsilon}^{\nu} \phi_{0}\right)(y) \mathbb{E}^{y}\left[f\left(X_{s-\varepsilon}\right) 1_{\{t-\varepsilon<\tau\}}\right] \nu(\mathrm{d} x) \mu(\mathrm{d} y) .
\end{aligned}
$$

With $f=1$ this implies

$$
\mathbb{P}^{\nu}(t<\tau)=\mathrm{e}^{-\lambda_{0} \varepsilon} \int_{M}\left(\psi_{\varepsilon}^{\nu} \phi_{0}\right)(y) \mathbb{P}^{y}(t-\varepsilon<\tau) \mu(\mathrm{d} y) \mu(\mathrm{d} y) .
$$

So, letting

$$
\nu_{\varepsilon}=\frac{\psi_{\varepsilon}^{\nu} \phi_{0}}{\mu\left(\psi_{\varepsilon}^{\nu} \phi_{0}\right)}=: h_{\varepsilon} \mu
$$

we arrive at

$$
\mathbb{E}^{\nu}\left[f\left(X_{s}\right) \mid t<\tau\right]=\frac{\mathbb{E}^{\nu}\left[f\left(X_{s}\right) 1_{\{t<\tau\}}\right]}{\mathbb{P}^{\nu}(t<\tau)}=\frac{\mathbb{E}^{\nu_{\varepsilon}}\left[f\left(X_{s-\varepsilon}\right) 1_{\{t-\varepsilon<\tau\}}\right]}{\mathbb{P}^{\nu_{\varepsilon}}(t-\varepsilon<\tau)}=\mathbb{E}^{\nu_{\varepsilon}}\left[f\left(X_{s-\varepsilon}\right) \mid t-\varepsilon<\tau\right] .
$$

Therefore,

$$
\mu_{t, \varepsilon}^{\nu}:=\frac{1}{t-\varepsilon} \int_{\varepsilon}^{t} \mathbb{E}^{\nu}\left(\delta_{X_{s}} \mid t<\tau\right) \mathrm{d} s=\mu_{t-\varepsilon}^{\nu_{\varepsilon}}, \quad t>\varepsilon .
$$

Since

$$
\mu\left(\psi_{\varepsilon}^{\nu} \phi_{0}\right)=\int_{M} p_{\varepsilon}^{0}(x, y) \phi_{0}(x) \phi_{0}(y) \nu(\mathrm{d} x) \mu(\mathrm{d} y)=\nu\left(\phi_{0} P_{\varepsilon}^{0} \phi_{0}^{-1}\right) \geq \nu\left(\phi_{0}\right)\left\|\phi_{0}\right\|_{\infty}^{-1}=: \alpha>0,
$$


by (2.9) we find a constant $c_{2}>0$ such that

$$
\left\|h_{\varepsilon} \phi_{0}^{-1}\right\|_{L^{2}\left(\mu_{0}\right)} \leq \alpha^{-1}\left\|\psi_{\varepsilon}^{\nu}\right\|_{L^{2}\left(\mu_{0}\right)} \leq \alpha^{-1}\left\|\phi_{0}\right\|_{\infty}\left\|p_{\varepsilon}^{0}\right\|_{L^{\infty}\left(\mu_{0}\right)} \leq c_{2} \varepsilon^{-\frac{d+2}{2}}, \quad \varepsilon \in(0,1) .
$$

Then (3.20) and (3.21) yield

$$
\begin{aligned}
& t^{2} \mathbb{W}_{2}\left(\mu_{t, \varepsilon}^{\nu}, \mu_{0}\right)^{2} \\
& \leq\left(1+\delta^{-1}\right) c_{1} c_{2} \alpha^{-1} t^{2} \mathrm{e}^{-\left(\lambda_{1}-\lambda_{0}\right) t} \varepsilon^{-\frac{d+2}{2}}+(1+\delta)\left(1+c t^{-1}\right) I_{\varepsilon}, \quad \delta>0, \varepsilon \in(0,1),
\end{aligned}
$$

where

$$
I_{\varepsilon}:=\frac{1}{\left\{\mu\left(\phi_{0}\right) \nu_{\varepsilon}\left(\phi_{0}\right)\right\}^{2}} \sum_{m=1}^{\infty} \frac{\left\{\nu_{\varepsilon}\left(\phi_{0}\right) \mu\left(\phi_{m}\right)+\mu\left(\phi_{0}\right) \nu_{\varepsilon}\left(\phi_{m}\right)\right\}^{2}}{\left(\lambda_{m}-\lambda_{0}\right)^{3}} .
$$

By (2.5), (2.6) and (3.2), we have

$$
\begin{aligned}
& \mu\left(\psi_{\varepsilon}^{\nu} \phi_{0}\right)=\nu\left(\phi_{0} P_{\varepsilon}^{-1} \phi_{0}^{-1}\right)=\mathrm{e}^{\lambda_{0} \varepsilon} \nu\left(P_{\varepsilon}^{D} 1\right), \\
& \mu\left(\psi_{\nu} \phi_{0}\right)=\nu\left(\phi_{0} P_{\varepsilon}^{0}\left(\phi_{m} \phi_{0}^{-1}\right)\right)=\mathrm{e}^{-\left(\lambda_{m}-\lambda_{0}\right) \varepsilon} \nu\left(\phi_{m}\right),
\end{aligned}
$$

so that

$$
\nu_{\varepsilon}\left(\phi_{m}\right)=\frac{\mathrm{e}^{-\lambda_{m} \varepsilon} \nu\left(\phi_{m}\right)}{\nu\left(P_{\varepsilon}^{D} 1\right)}, \quad m \geq 0 .
$$

Thus, $\lim _{\varepsilon \rightarrow 0} \nu_{\varepsilon}\left(\phi_{0}\right)=\nu\left(\phi_{0}\right)$ and there exists a constant $C>1$ such that

$$
C^{-1} \mathrm{e}^{-\lambda_{m} \varepsilon}\left|\nu\left(\phi_{m}\right)\right| \leq\left|\nu_{\varepsilon}\left(\phi_{m}\right)\right| \leq C\left|\nu\left(\phi_{m}\right)\right|, \quad m \geq 1, \varepsilon \in(0,1) .
$$

Therefore, if $I<\infty$, by this and

$$
\sum_{m=1}^{\infty} \mu\left(\phi_{m}\right)^{2} \leq \mu(1)=1
$$

we may apply the dominated convergence theorem to derive $\lim _{\varepsilon \rightarrow 0} I_{\varepsilon}=I$. On the other hand, if $I=\infty$, which is equivalent to

$$
\sum_{m=1}^{\infty} \frac{\nu\left(\phi_{m}\right)^{2}}{\left(\lambda_{m}-\lambda_{0}\right)^{3}}=\infty
$$

then by (3.24) and the monotone convergence theorem we get

$$
\liminf _{\varepsilon \rightarrow 0} \sum_{m=1}^{\infty} \frac{\nu_{\varepsilon}\left(\phi_{m}\right)^{2}}{\left(\lambda_{m}-\lambda_{0}\right)^{3}} \geq C^{-2} \liminf _{\varepsilon \rightarrow 0} \sum_{m=1}^{\infty} \frac{e^{-2 \lambda_{m} \varepsilon} \nu\left(\phi_{m}\right)^{2}}{\left(\lambda_{m}-\lambda_{0}\right)^{3}}=\infty,
$$

which together with (3.25) and $\nu_{\varepsilon}\left(\phi_{0}\right) \rightarrow \nu\left(\phi_{0}\right)$ implies

$$
\liminf _{\varepsilon \rightarrow 0} I_{\varepsilon}=\frac{1}{\left\{\mu\left(\phi_{0}\right) \nu\left(\phi_{0}\right)\right\}^{2}} \liminf _{\varepsilon \rightarrow 0} \sum_{m=1}^{\infty} \frac{\left\{\nu_{\varepsilon}\left(\phi_{0}\right) \mu\left(\phi_{m}\right)+\mu\left(\phi_{0}\right) \nu_{\varepsilon}\left(\phi_{m}\right)\right\}^{2}}{\left(\lambda_{m}-\lambda_{0}\right)^{3}}
$$




$$
\geq \frac{1}{\left\{\mu\left(\phi_{0}\right) \nu\left(\phi_{0}\right)\right\}^{2}} \liminf _{\varepsilon \rightarrow 0} \frac{\frac{1}{2}\left\{\mu\left(\phi_{0}\right) \nu_{\varepsilon}\left(\phi_{m}\right)\right\}^{2}-\left\|\phi_{0}\right\|_{\infty}^{2} \mu\left(\phi_{m}\right)^{2}}{\left(\lambda_{m}-\lambda_{0}\right)^{3}}=\infty .
$$

In conclusion, we have

$$
\lim _{\varepsilon \rightarrow 0} I_{\varepsilon}=I
$$

This together with (3.23) for $\varepsilon=t^{-2}$ gives

$$
\limsup _{t \rightarrow \infty}\left\{t^{2} \mathbb{W}_{2}\left(\mu_{t, t^{-2}}^{\nu}, \mu_{0}\right)^{2}\right\} \leq I \text {. }
$$

On the other hand, it is easy to see that

$$
\left\|\mu_{t, \varepsilon}^{\nu}-\mu_{t}^{\nu}\right\|_{v a r} \leq \frac{2 \varepsilon}{t}, \quad 0<\varepsilon<t
$$

so that

$$
\mathbb{W}_{2}\left(\mu_{t}^{\nu}, \mu_{t, t^{-2}}^{\nu}\right)^{2} \leq D^{2}\left\|\mu_{t, t^{-2}}^{\nu}-\mu_{t}^{\nu}\right\|_{v a r} \leq 2 D^{2} t^{-3}, \quad t>1 .
$$

Combining this with (3.27), we prove (3.18).

\section{Lower bound estimate and the finiteness of the limit}

We will follow the idea of [1, 18], for which we need to modify $\tilde{\mu}_{t}^{\nu}$ as follows. For any $\beta>0$, consider

$$
\tilde{\mu}_{t, \beta}^{\nu}:=\left(1+\tilde{\rho}_{t, \beta}^{\nu}\right) \mu_{0}, \quad \tilde{\rho}_{t, \beta}^{\nu}:=P_{t^{-\beta}}^{0} \tilde{\rho}_{t}^{\nu}, \quad t>0 .
$$

According to Lemma 3.2, there exists $t_{0}>0$ such that

$$
\tilde{h}_{t}^{\nu}:=1+\tilde{\rho}_{t}^{\nu} \geq \frac{1}{2}, \quad \tilde{h}_{t, \beta}^{\nu}:=1+\tilde{\rho}_{t, \beta}^{\nu} \geq \frac{1}{2}, \quad \beta>0, t \geq t_{0} .
$$

Consequently, $\tilde{\mu}_{t, \beta}^{\nu}$ and $\tilde{\mu}_{t}^{\nu}$ are probability measures for any $\beta>0, t \geq t_{0}$.

Lemma 4.1. For any $\beta>0$, there exists a constant $c>0$ such that $f_{t, \beta}:=L_{0}^{-1} \tilde{\rho}_{t, \beta}^{\nu}$ satisfies

$$
\left\|f_{t, \beta}\right\|_{\infty}+\left\|L_{0} f_{t, \beta}\right\|_{\infty}+\left\|\nabla f_{t, \beta}\right\|_{\infty} \leq c t^{\frac{5 \beta d}{4}-1}, \quad t \geq 1 .
$$

Proof. By (2.6) and (3.8), we have

$$
\begin{aligned}
& f_{t, \beta}=-\sum_{m=1}^{\infty} \frac{\left\{\mu\left(\phi_{0}\right) \nu\left(\phi_{m}\right)+\nu\left(\phi_{0}\right) \mu\left(\phi_{m}\right)\right\} \mathrm{e}^{-\left(\lambda_{m}-\lambda_{0}\right) t^{-\beta}}}{t\left(\lambda_{m}-\lambda_{0}\right)^{2} \nu\left(\phi_{0} P_{t}^{0} \phi_{0}^{-1}\right)}\left(\phi_{m} \phi_{0}^{-1}\right), \\
& L_{0} f_{t, \beta}=\sum_{m=1}^{\infty} \frac{\left\{\mu\left(\phi_{0}\right) \nu\left(\phi_{m}\right)+\nu\left(\phi_{0}\right) \mu\left(\phi_{m}\right)\right\} \mathrm{e}^{-\left(\lambda_{m}-\lambda_{0}\right) t^{-\beta}}}{t\left(\lambda_{m}-\lambda_{0}\right) \nu\left(\phi_{0} P_{t}^{0} \phi_{0}^{-1}\right)}\left(\phi_{m} \phi_{0}^{-1}\right) .
\end{aligned}
$$


Combining these with (2.1), (2.12), (3.5), and

$$
\left|\mu\left(\phi_{0}\right) \nu\left(\phi_{m}\right)+\nu\left(\phi_{0}\right) \mu\left(\phi_{m}\right)\right| \leq\left\|\phi_{0}\right\|_{\infty}+\left\|\phi_{m}\right\|_{\infty} \preceq m, \quad m \geq 1,
$$

we find a constant $c_{1}>0$ such that

$$
\begin{aligned}
& t\left\{\left\|f_{t, \beta}\right\|_{\infty}+\left\|L_{0} f_{t, \beta}\right\|_{\infty}\right\} \preceq \sum_{m=1}^{\infty} \frac{\mathrm{e}^{-\left(\lambda_{m}-\lambda_{0}\right) t^{-\beta}} m^{\frac{3 d+2}{2 d}}}{\lambda_{m}-\lambda_{0}} \\
& \preceq \sum_{m=1}^{\infty} \mathrm{e}^{-c_{1} m^{\frac{2}{d} t^{-\beta}}} m^{\frac{3 d-2}{2 d}} \preceq \int_{0}^{\infty} \mathrm{e}^{-c_{1} s^{\frac{2}{d}} t^{-\beta}} s^{\frac{3 d-2}{2 d}} \mathrm{~d} s \preceq t^{\frac{\beta(5 d-2)}{4}}, \quad t \geq 1 .
\end{aligned}
$$

Similarly, (2.25) implies

$$
\begin{aligned}
& t\left\|\nabla f_{t, \beta}\right\|_{\infty} \preceq \sum_{m=1}^{\infty} \frac{\mathrm{e}^{-\left(\lambda_{m}-\lambda_{0}\right) t^{-\beta}} m^{\frac{3 d+4}{2 d}}}{\left(\lambda_{m}-\lambda_{0}\right)^{2}} \\
& \preceq \sum_{m=1}^{\infty} \mathrm{e}^{-c_{1} m^{\frac{2}{d}} t^{-\beta}} m^{\frac{3 d-4}{2 d}} \preceq t^{\frac{\beta(5 d-4)}{4}}, \quad t \geq 1 .
\end{aligned}
$$

Then the proof is finished.

Lemma 4.2. For any $\beta \in\left(0, \frac{1}{20 d}\right]$, there exits a constant $c>0$ such that

$$
t^{2} \mathbb{W}_{2}\left(\tilde{\mu}_{t, \beta}^{\nu}, \mu_{0}\right)^{2} \geq \frac{1-c t^{-1}}{\left\{\mu\left(\phi_{0}\right) \nu\left(\phi_{0}\right)\right\}^{2}} \sum_{m=1}^{\infty} \frac{\left\{\mu\left(h \phi_{0}\right) \mu_{0}\left(\phi_{m}\right)+\mu\left(\phi_{0}\right) \nu\left(\phi_{m}\right)\right\}^{2}}{\left(\lambda_{m}-\lambda_{0}\right)^{3}}-c t^{-\frac{1}{4}} .
$$

Proof. To estimate $\mathbb{W}_{2}\left(\tilde{\mu}_{t, \beta}^{\nu}, \mu_{0}\right)$ from below by using the argument in [1, 18], we take

$$
\varphi_{\theta}^{\varepsilon}:=-\varepsilon \log P_{\frac{\varepsilon \theta}{2}}^{0} \mathrm{e}^{-\varepsilon^{-1} f_{t, \beta}}, \quad \theta \in[0,1], \varepsilon>0 .
$$

We have $\varphi_{0}^{\varepsilon}=f_{t, \beta},\left\|\varphi_{\theta}^{\varepsilon}\right\|_{\infty} \leq\left\|f_{t, \beta}\right\|_{\infty}$, and by [18, Lemma 2.9], there exists a constant $c_{1}>0$ such that for any $\varepsilon \in(0,1)$,

$$
\begin{aligned}
& \varphi_{1}^{\varepsilon}(y)-\varphi_{0}^{\varepsilon}(x) \leq \frac{1}{2}\left\{\rho(x, y)^{2}+\varepsilon\left\|\left(L_{0} f_{t, \beta}\right)^{+}\right\|_{\infty}+c_{1} \sqrt{\varepsilon}\left\|\nabla f_{t, \beta}\right\|_{\infty}^{2}\right\}, \quad x, y \in M, \\
& \int_{M}\left(\varphi_{0}^{\varepsilon}-\varphi_{1}^{\varepsilon}\right) \mathrm{d} \mu_{0} \leq \frac{1}{2} \int_{M}\left|\nabla f_{t, \beta}\right|^{2} \mathrm{~d} \mu_{0}+c_{1} \varepsilon^{-1}\left\|\nabla f_{t, \beta}\right\|_{\infty}^{4} .
\end{aligned}
$$

Therefore, by the Kantorovich dual formula, $\varphi_{0}^{\varepsilon}=f_{t, \beta}$ and the integration by parts formula

$$
\int_{M} f_{t, \beta} \tilde{\rho}_{t, \beta}^{\nu} \mathrm{d} \mu_{0}=\int_{M} f_{t, \beta} L_{0} f_{t, \beta} \mathrm{d} \mu_{0}=-\int_{M}\left|\nabla f_{t, \beta}\right|^{2} \mathrm{~d} \mu_{0},
$$

we find a constant $c>0$ such that

$$
\begin{aligned}
& c\left(\varepsilon\left\|L_{0} f_{t, \beta}\right\|_{\infty}+\varepsilon^{\frac{1}{2}}\left\|\nabla f_{t, \beta}\right\|_{\infty}^{2}\right)+\frac{1}{2} \mathbb{W}_{2}\left(\tilde{\mu}_{t, \beta}^{\nu}, \mu_{0}\right)^{2} \geq \int_{M} \varphi_{1}^{\varepsilon} \mathrm{d} \mu_{0}-\int_{M} \varphi_{0}^{\varepsilon} \mathrm{d} \tilde{\mu}_{t, \beta}^{\nu} \\
& =\int_{M}\left(\varphi_{1}^{\varepsilon}-\varphi_{0}^{\varepsilon}\right) \mathrm{d} \mu_{0}-\int_{M} f_{t, \beta} \tilde{\rho}_{t, \beta}^{\nu} \mathrm{d} \mu_{0}=\int_{M}\left(\varphi_{1}^{\varepsilon}-\varphi_{0}^{\varepsilon}\right) \mathrm{d} \mu_{0}-\int_{M} f_{t, \beta} L_{0} f_{t, \beta} \mathrm{d} \mu_{0} \\
& \geq \frac{1}{2} \int_{M}\left|\nabla f_{t, \beta}\right|^{2} \mathrm{~d} \mu_{0}-c \varepsilon^{-1}\left\|\nabla f_{t, \beta}\right\|_{\infty}^{4} .
\end{aligned}
$$


Taking $\varepsilon=t^{-\frac{3}{2}}$ and applying Lemma 4.1, when $\beta \leq \frac{1}{20 d}$ we find a constant $c^{\prime}>0$ such that

$$
t^{2} \mathbb{W}_{2}\left(\tilde{\mu}_{t, \beta}^{\nu}, \mu_{0}\right)^{2} \geq t^{2} \mu_{0}\left(\left|\nabla f_{t, \beta}\right|^{2}\right)-c^{\prime} t^{-\frac{1}{4}}, \quad t \geq t_{0}
$$

Combining this with (3.5) and (4.3), we complete the proof.

Lemma 4.3. There exist constants $c, t_{0}>0$ such that for any $\nu=h \mu \in \mathscr{P}_{0}$ with $h \in L^{2}(\mu)$, $\tilde{\mu}_{t, \beta}^{\nu}, \tilde{\mu}_{t}^{\nu} \in \mathscr{P}_{0}$ for $t \geq t_{0}$ and

$$
t \mathbb{W}_{2}\left(\tilde{\mu}_{t, \beta}^{\nu}, \tilde{\mu}_{t}^{\nu}\right) \leq c\|h\|_{L^{2}(\mu)} t^{-\beta}, \quad t \geq t_{0} .
$$

Proof. $\tilde{\mu}_{t, \beta}^{\nu}, \tilde{\mu}_{t}^{\nu} \in \mathscr{P}_{0}$ for large $t$ is implied by Lemma 3.2. Next, by (4.1), we have

$$
\mathscr{M}\left(\tilde{h}_{t}^{\nu}, \tilde{h}_{t, \beta}^{\nu}\right) \geq \frac{1}{2}
$$

so that [18, Lemma 2.3] implies

$$
\mathbb{W}_{2}\left(\tilde{\mu}_{t, \beta}^{\nu}, \tilde{\mu}_{t}^{\nu}\right)^{2} \leq \int_{M} \frac{\left|\nabla L_{0}^{-1}\left(\tilde{h}_{t}^{\nu}-\tilde{h}_{t, \beta}^{\nu}\right)\right|^{2}}{\mathscr{M}\left(\tilde{h}_{t}^{\nu}, \tilde{h}_{t, \beta}^{\nu}\right)} \mathrm{d} \mu_{0} \leq 2 \mu_{0}\left(\left|\nabla L_{0}^{-1}\left(\tilde{\rho}_{t}^{\nu}-\tilde{\rho}_{t, \beta}^{\nu}\right)\right|^{2}\right) .
$$

To estimate the upper bound in this inequality, we first observe that by (3.7) and (3.8), when $\nu=h \mu$ we have

$$
\begin{aligned}
& L_{0}^{-1}\left(\tilde{\rho}_{t, \beta}^{\nu}-\tilde{\rho}_{t}^{\nu}\right)=L_{0}^{-1}\left(P_{t^{-\beta}}^{0} \tilde{\rho}_{t}^{\nu}-\tilde{\rho}_{t}^{\nu}\right)=\int_{0}^{t^{-\beta}} P_{r}^{0} \tilde{\rho}_{t}^{\nu} \mathrm{d} r \\
& =\frac{1}{t \nu\left(\phi_{0} P_{t}^{0} \phi_{0}^{-1}\right)} \int_{0}^{t^{-\beta}}\left(-L_{0}\right)^{-1}\left(P_{r}^{0}-\mu_{0}\right) g \mathrm{~d} r
\end{aligned}
$$

where

$$
g:=\mu\left(\phi_{0}\right) h \phi_{0}^{-1}+\nu\left(\phi_{0}\right) \phi_{0}^{-1}
$$

Since $\|h\|_{L^{2}(\mu)} \geq \mu(h)=1$,

$$
\|g\|_{L^{2}\left(\mu_{0}\right)} \leq\left\|\phi_{0}\right\|_{\infty}\left(1+\|h\|_{L^{2}(\mu)}\right) \leq 2\left\|\phi_{0}\right\|_{\infty}\|h\|_{L^{2}(\mu)} .
$$

By (2.10), (4.6) and the fact that $\left(-L_{0}\right)^{-\frac{1}{2}}=c \int_{0}^{\infty} P_{s^{2}}^{0} \mathrm{~d} s$ for some constant $c>0$, we find a constants $c_{1}, c_{2}>0$ such that

$$
\begin{aligned}
& \left\|\nabla L_{0}^{-1}\left(P_{r}^{0}-\mu_{0}\right) g\right\|_{L^{2}\left(\mu_{0}\right)}=\left\|L_{0}^{-\frac{1}{2}}\left(P_{r}^{0}-\mu_{0}\right) g\right\|_{L^{2}\left(\mu_{0}\right)} \leq \int_{0}^{\infty}\left\|\left(P_{r+s^{2}}^{0}-\mu_{0}\right) g\right\|_{L^{2}\left(\mu_{0}\right)} \mathrm{d} s \\
& \leq c_{1}\|h\|_{L^{2}(\mu)} \int_{1}^{\infty} \mathrm{e}^{-\left(\lambda_{1}-\lambda_{0}\right)\left(s^{2}+r\right)} \mathrm{d} s \leq c_{2}\|h\|_{L^{2}(\mu)}, \quad r \in[0,1] .
\end{aligned}
$$

Therefore, by (3.5) and (4.5), we obtain

$$
\left\|\nabla L_{0}^{-1}\left(\tilde{\rho}_{t, \beta}^{\nu}-\tilde{\rho}_{t}^{\nu}\right)\right\|_{L^{2}\left(\mu_{0}\right)} \preceq \frac{1}{t} \int_{0}^{t^{-\beta}}\left\|\nabla L_{0}^{-1}\left(P_{r}^{0}-\mu_{0}\right) g\right\|_{L^{2}\left(\mu_{0}\right)} \mathrm{d} r \preceq t^{-(1+\beta)}\|h\|_{L^{2}(\mu)}, \quad t \geq t_{0} .
$$

Combining this with (4.4) we finish the proof. 
We are now ready to prove the following result.

Proposition 4.4. For any $\nu \in \mathscr{P}_{0}$,

$$
\liminf _{t \rightarrow \infty}\left\{t^{2} \mathbb{W}_{2}\left(\mu_{t}^{\nu}, \mu_{0}\right)^{2}\right\} \geq I>0
$$

and $I<\infty$ provided either $d \leq 7$, or $d \geq 7$ but $\nu=h \mu$ with $h \in L^{\frac{2 d}{d+6}}$.

Proof. Let $\beta \in\left(0, \frac{1}{20 d}\right]$. By (3.19), Lemma 4.2 and Lemma 4.3, there exist constants $c, t_{0}>0$ such that for $\nu=h \mu \in \mathscr{P}_{0}$ and $t \geq t_{0}$,

$$
\begin{aligned}
& t \mathbb{W}_{2}\left(\mu_{t}^{\nu}, \tilde{\mu}_{t}^{\nu}\right) \leq c\|h\|_{L^{2}(\mu)} t^{-\beta t}, \\
& t \mathbb{W}_{2}\left(\tilde{\mu}_{t, \beta}^{\nu}, \mu_{0}\right) \geq\left(\left\{\left(1-c t^{-1}\right) I-c t^{-\frac{1}{4}}\right)^{+}\right\}^{\frac{1}{2}}, \\
& t \mathbb{W}_{2}\left(\mu_{t}^{\nu}, \tilde{\mu}_{t}^{\nu}\right) \leq c t \mathrm{e}^{-\left(\lambda_{1}-\lambda_{0}\right) t / 2}\|h\|_{L^{2}(\mu)}^{\frac{1}{2}} .
\end{aligned}
$$

Then

$$
t \mathbb{W}_{2}\left(\mu_{t}^{\nu}, \mu_{0}\right) \geq\left(\left\{\left(1-c t^{-1}\right) I-c t^{-\frac{1}{4}}\right)^{+}\right\}^{\frac{1}{2}}-c\|h\|_{L^{2}(\mu)} t^{-\beta t}-c t \mathrm{e}^{-\left(\lambda_{1}-\lambda_{0}\right) t / 2}\|h\|_{L^{2}(\mu)}^{\frac{1}{2}}, \quad t \geq t_{0}
$$

In general, let $\mu_{t, \varepsilon}^{\nu}=\mu_{t-\varepsilon}^{\nu_{\varepsilon}}$ be as in the proof of Proposition 3.4. Applying (4.8) to $\mu_{t, t^{-2}}^{\nu}$ replacing $\mu_{y}^{\nu}$ and using (3.22), (3.26), we obtain

$$
\liminf _{t \rightarrow \infty}\left\{t \mathbb{W}_{2}\left(\mu_{t, t^{-2}}^{\nu}, \mu_{0}\right)\right\} \geq \sqrt{I}
$$

which together with (3.28) proves (4.7).

It remains to prove $I>0$ and $I<\infty$ the under given conditions, where due to (3.25), $I<\infty$ is equivalent to

$$
I^{\prime}:=\sum_{m=1}^{\infty} \frac{\nu\left(\phi_{m}\right)^{2}}{\lambda_{m}^{3}}<\infty
$$

Below we first prove $I>0$ then show $I^{\prime}<\infty$ under the given conditions.

(a) $I>0$. If this is not true, then

$$
\mu\left(h \phi_{0}\right) \mu\left(\phi_{m}\right)=-\mu\left(\phi_{0}\right) \mu\left(h \phi_{m}\right), \quad m \geq 1 .
$$

Combining this with the representation in $L^{2}(\mu)$

$$
f=\sum_{m=0}^{\infty} \mu\left(f \phi_{m}\right) \phi_{m}, \quad f \in L^{2}(\mu)
$$

where the equation holds point-wisely if $f \in C_{b}(M)$ by the continuity, we obtain

$$
\mu\left(\phi_{0}\right) \nu(f)=\sum_{m=0}^{\infty} \mu\left(f \phi_{m}\right) \mu\left(\phi_{0}\right) \nu\left(\phi_{m}\right)=2 \mu\left(f \phi_{0}\right) \nu\left(\phi_{0}\right) \mu\left(\phi_{0}\right)-\sum_{m=0}^{\infty} \mu\left(f \phi_{m}\right) \mu\left(\phi_{m}\right) \nu\left(\phi_{0}\right)
$$




$$
=2 \mu\left(f \phi_{0}\right) \nu\left(\phi_{0}\right) \mu\left(\phi_{0}\right)-\nu\left(\phi_{0}\right) \mu(f), \quad f \in C_{b}(M)
$$

Consequently,

$$
0 \leq \mu\left(\phi_{0}\right) \frac{\mathrm{d} \nu}{\mathrm{d} \mu}=2 \phi_{0} \nu\left(\phi_{0}\right) \mu\left(\phi_{0}\right)-\nu\left(\phi_{0}\right),
$$

which is however impossible since the upper bound is negative in a neighborhood of $\partial M$, because $\nu\left(M^{\circ}\right)>0$ implies $\nu\left(\phi_{0}\right)>0$ for $\phi_{0}>0$ in $M^{\circ}$, and $\phi_{0}$ is continuous with $\left.\phi_{0}\right|_{\partial M}=0$. Therefore, we must have $I>0$.

(b) $I^{\prime}<\infty$. Let $\left\{h_{n}\right\}_{n \geq 1}$ be a sequence of probability density functions with respect to $\mu$ such that

$$
\nu_{n}:=h_{n} \mu \rightarrow \nu \text { weakly as } n \rightarrow \infty
$$

By the spectral representation for $(-L)^{-\frac{3}{2}}$, and applying the Sobolev inequality (1.2) with $p=\frac{2 d}{d+6} \vee 1$, we obtain

$$
I_{n}^{\prime}:=\sum_{m=1}^{\infty} \frac{\nu_{n}\left(\phi_{m}\right)^{2}}{\lambda_{m}^{3}} \leq\left\|(-L)^{-\frac{3}{2}} h_{n}\right\|_{L^{2}(\mu)}^{2} \leq K^{2}\left\|h_{n}\right\|_{L^{\frac{2 d}{d+6} \vee 1}(\mu)}^{2}, \quad n \geq 1 .
$$

It is easy to see that for $d \leq 6$ we have $\frac{2 d}{d+6} \leq 1$, so that $\left\|h_{n}\right\|_{L^{\frac{2 d}{d+6} \vee 1}(\mu)}=\mu\left(h_{n}\right)=1$. Combining this with (4.10), (4.11) and applying Fatou's lemma, we derive

$$
I^{\prime}=\sum_{m=1}^{\infty} \liminf _{n \rightarrow \infty} \frac{\nu_{n}\left(\phi_{m}\right)^{2}}{\lambda_{m}^{3}} \leq \liminf _{n \rightarrow \infty} I_{n}^{\prime} \leq K^{2}<\infty, \quad d \leq 6 .
$$

Finally, when $d \geq 7$ and $\nu=h \mu$ with $h \in L^{\frac{2 d}{d+6}}(\mu)$, by applying (4.11) to $h_{n}=h$ we prove $I^{\prime}<\infty$.

Acknowledgement. The author would like to thank the referee for helpful comments and corrections.

\section{References}

[1] L. Ambrosio, F. Stra, D. Trevisan, A PDE approach to a 2-dimensional matching problem, Probab. Theory Relat. Fields 173(2019), 433-477.

[2] D. Bakry, M. Emery, Hypercontractivité de semi-groupes de diffusion, C. R. Acad. Sci. Paris. Sér. I Math. 299(1984), 775-778.

[3] D. Bakry, I. Gentil, M. Ledoux, Analysis and Geometry of Markov Diffusion Operators, Springer, 2014.

[4] I. Chavel, Eigenvalues in Riemannian Geometry, Academic Press, 1984. 
[5] J. Chen, S. Jian, A remark on quasi-ergodicity of ultracontractive Markov processes, Statis. Probab. Letters 87(2014), 184-190.

[6] P. Collet, S. Martinez, J. San Martin, Quasi-Stationary Distributions, Springer, 2013.

[7] E. B. Davies, Heat Kernels and Spectral Theory, Cambridge University Press, 1989.

[8] E. M. Ouhabaz, Analysis of Heat Equations on Domains, London Mathematical Society, 2005.

[9] E. M. Ouhabaz, F.-Y. Wang, Sharp estimates for intrinsic ultracontractivity on $C^{1, \alpha}{ }_{-}$ domains, Manuscripta Math. 122(2007), 229-244.

[10] F.-Y. Wang, Functional inequalities, semigroup properties and spectrum estimates, Infinite Dimensional Analysis, Quantum Probability and Related Topics 3:2(2000), 263295.

[11] F.-Y. Wang, Functional Inequalities, Markov Semigroups and Spectral Theory, Science Press, 2005.

[12] F.-Y. Wang, Estimates of the first Neumann eigenvalue and the log-Sobolev constant on Non-convex manifolds, Math. Nach. 280(2007), 1431-1439.

[13] F.-Y. Wang, Analysis for Diffusion Processes on Riemnnian Manifolds, Springer, 2014.

[14] F.-Y. Wang, Modified curvatures on manifolds with boundary and applications, Pot. Anal. 41(2014), 699-714.

[15] F.-Y. Wang, Convergence in Wasserstein distance for empirical measures of Dirichlet diffusion processes on manifolds, arXiv:2005.09290.

[16] F.-Y. Wang, Wasserstein convergence rate for empirical measures on noncompact manifolds, arXiv:2007.14667.

[17] F.-Y. Wang, Convergence in Wasserstein distance for empirical measures of semilinear SPDEs, arXiv:2102.00361.

[18] F.-Y. Wang, J.-X. Zhu, Limit theorems in Wasserstein distance for empirical measures of diffusion processes on Riemannian manifolds, aXiv:1906.03422. 